\title{
DIALYSIS CATHETER PLACEMENT: RETENTION OF A GUIDE WIRE
}

Prasad Kulkarni ${ }^{1}$, Naga Shiva Karthik Bhandaru², R. Shivaraman ${ }^{3}$

\section{HOW TO CITE THIS ARTICLE:}

Prasad Kulkarni, Naga Shiva Karthik Bhandaru, R. Shivaraman. "Dialysis Catheter Placement: Retention of a Guide Wire". Journal of Evolution of Medical and Dental Sciences 2014; Vol. 3, Issue 48, September 29;

Page: $11622-11625$, DOI: $10.14260 /$ jemds/2014/3525

ABSTRACT: Central venous catheterization is a commonly performed procedure in the intensive care unit. Guide wires are routinely used in the Seldinger technique during central venous catheter placement. We present a case in which a guide wire was retained during placement of double lumen dialysis catheter and how we expeditiously managed the scenario.

KEYWORDS: Seldinger technique, Double- lumen Dialysis catheter, Guide Wire.

INTRODUCTION: Central venous catheterization is a commonly performed procedure in the intensive care unit that is technically challenging, and associated with several risks and complications. Guide wires are routinely used in the Seldinger technique during central venous catheter placement. ${ }^{1}$ Catheter looping and knotting are known complications of central venous catheterization. The most common known complication of a guide wire is cardiac arrhythmias. Other complications also include looping and knotting. Although intravascular entrapment of a guide wire is rare, this complication needs to be emphasized as it increases morbidity and mortality. We present a case in which a guide wire was retained during placement of double lumen dialysis cathetre through internal jugular vein.

CASE REPORT: Here we are reporting a case 28 year old female who came to our hospital with complaints of fever for 4 days and cough with breathlessness for 2 days. She was evaluated in Emergency room. In view of respiratory distress and impending respiratory failure with nonreassuring oxygen saturation, she was intubated and mechanical ventilation instituted. She was shifted to Intensive care unit. The initial history, clinical examination and laboratory investigations hinted a working diagnosis of septicaemic shock with metabolic acidosis and pre renal azotemia. She was planned for emergency Hemodialysis in view of above clinical condition with sr. creatinine of $8 \mathrm{mg} / \mathrm{dl}$. Past history did not reveal any evidence of chronic renal insufficiency.

A double lumen dialysis catheter 11.5 Fr, was planned to insert in Right internal jugular vein through seldinger technique. After puncturing vein and aspiration of venous blood, guide wire was threaded and needle removed. Dilation of skin tract done. Cathetre could not be negotiated beyond subcutaneous tissue. Malposition of guide wire suspected. Guide wire was pulled out but could not be withdrawn out. Kinking of guide wire was suspected. Guide wire was left in-situ and sterile occlusive dressing done. We proceeded with alternate site, left femoral vein, for hemodialysis.

A dual lumen catheter was placed successfully using seldinger technique. Hemodialysis was instituted. Patient was stable throughout the procedure. An emergency chest - roentengenogram revealed looping of guide wire in right side neck in subcutaneous plane. Consultation with Surgical faculty was obtained. A trial of pulling out guide wire with gentle traction was suggested with backup plan for thoracotomy and removal of foreign body. Surprisingly, the guide wire has been pulled out in to and mangled portion was observed. 
Suspicion of vessel puncture mandated us to do repeat chest -roentgenogram. Patient was hemodynamically stable and no evidence of enlarging subcutaneous mass. Chest -roentgenogram revealed normal anatomy and no distortion of subcutaneous plane. She was observed for 24 hours. It was uneventful. Repeat laboratory investigation revealed normal blood count and serum creatinine was $4 \mathrm{mg} / \mathrm{dl}$ and improvement of metabolic acidosis.

C.T. Chest was planned for excluding vascular damage. Citing personal reasons and even after through counseling, patient attendees requested for discharge against medical advice. All supports were withdrawn and patient was shifted to their personal transport.

DISCUSSION: Double lumen catheterization is utilized for emergency dialysis. Complications such as looping, knotting, vascular perforation, fragmentation and displacement of guide wire are encountered frequently. ${ }^{2}$ Catheter-related complications are well known, but there are few reports in which a guide wire has been involved.

Common guide wire-related complications reported are entrapment of guide wire in the sternomastoid muscle and inferior vena cava filters. ${ }^{3-5}$ Guide wire retention during central venous catheterization is not unheard of. In Western literature there are case reports of retained catheters and guide wires, making them the most commonly reported non-surgically retained objects. ${ }^{6-8}$

The management of misplaced guide wires will depend on the location and, indication for central access, and clinical condition of the patient. If there is a suspicion that a guide wire is misplaced then there must be further consideration before removal, due to the risk of complications, in particular uncontrolled haemorrhage.

In the short term, it is generally safer to leave the device in situ and consult avascular surgeon or interventional radiologist rather than a hasty removal with pressure applied to the access site. This case serves to raise awareness for invasive procedure protocol in terms of accountability for all utilized equipment, even in bedside procedures, as well as a systematic analysis of routine radiographs, accounting for all man-made objects.

Migration of a guide wire into the circulation can occur from any of the usual CV catheter insertion sites. ${ }^{9-12}$ A complete guide wire may not necessarily produce any symptoms and its loss may remain unnoticed for long. ${ }^{10}$ However, intravascular migration of a broken guide wire has the potential of causing adverse effects like vascular damage, thrombosis, embolism and arrhythmias; embolism from guide wire fragments can be fatal in up to $20 \%$ instances.

Cardiac tamponade manifesting 3 years after a guide wire loss has been reported as a late complication, highlighting the importance of wire extraction as soon as a diagnosis is made. ${ }^{11}$ Retrieval is usually done by interventional radiology using gooseneck snares, endovascular retrieval forceps or Dormia baskets; surgical removal is also reported. After the occurrence of this complication, we made some changes in the dialysis catheter insertion protocol.

Force should not be applied during insertion/withdrawal of the guide wire. Any resistance felt should prompt removal of needle and guide wire en-bloc. In our case the initial false positive aspiration of blood was probably due to bleed from subcutaneous vessel and subsequently forcing of guide wire could have caused the knotting in the subcutaneous plane. This might account for the intactness of vascular structure in our patient. 
To ensure the removal of a guide wire after insertion, the following recommendations should be strictly followed:

- The physician should be aware of the risk factors involved in the procedure.

- The trainee, supervisor or assistant must hold the wire at all times.

- The physician should be reminded of guide wire removal.

- Besides an insertion checklist and documentation, there must be an observer who verifies removal of the guide wire.

- The physician should complete theoretical and practical training in inserting and removing central lines. ${ }^{12}$

\section{REFERENCES:}

1. Huang CC, Chen JH, Huang $\mathrm{HH}$, Yen DH, Kao WF, Huang CI, et al. Emergency femoral hemodialysis catheter placement complicated by prevesical hematoma. J Emerg Med. 2010; 39: $583-5$

2. Katiyar S, Jain RK. Entrapped central venous catheter guide wire. Indian J Anaesth. 2010; 54: 354-5.

3. Mastan M, Clothier PR, Ousta B, Deulkar U. Internal jugular venous cannulation complicated by J-tip guide wire entrapment. Br J Anaesth. 2001; 86: 292-3.

4. Polos PG, Sahn SA. Complication of central venous catheter insertion: Fragmentation of a guidewire with pulmonary artery embolism. Crit Care Med. 1991; 19: 438-40.

5. Andrews RT, Gesch wind JF, Savadar SJ, Venbrux AC. Entrapment of J-tip guide wires by venetech and stainless-steel Greenfield vena cava filters during central venous catheter placement. Percutaneous management in four patients. Cardiovasc Intervent Radiol. 1998; 21: 424-8.

6. Sznajder JI, Zveibil FR, Bitterman H, Weiner P, Bursztein S. Central vein catheterization: failure and complication rates by three percutaneous approaches. Arch Intern Med. 1986; 146: 25961.

7. Mansfield PF, Hohn DC, Fornage BD, Gregurich MA, Ota DM. Complications and failures of subclavian-vein catheterization. N Engl J Med. 1994; 331: 1735-8.

8. Quality \& Safety. A Newsletter from the New York State Department of Health. Office of Health Systems Management. Winter 2011; 5(1): 1-10.

9. Schummer W, Schummer C, Gaser E, Bartunek R. Loss of the guide wire: mishap or blunder? BJA. 2002; 88: 144-6.

10. Guo H, Peng F, Ueda T. Loss of the guide wire: a case report. Circ J. 2006; 70: 1520-2.

11. Pérez-Díez D, Salgado-Fernández J, Vázquez-González N, Calviño-Santos R, Vázquez-Rodríguez JM, Aldama - López G, et al. Percutaneous retrieval of a lost guide wire that caused cardiac tamponade. Circulation. 2007; 115: 629-31.

12. New South Wales - National Trust of Australia. NSW Government. Health. Safety Alert 002/09 Guide wire Alert (2009). June 16, 2009. 


\section{CASE REPORT}

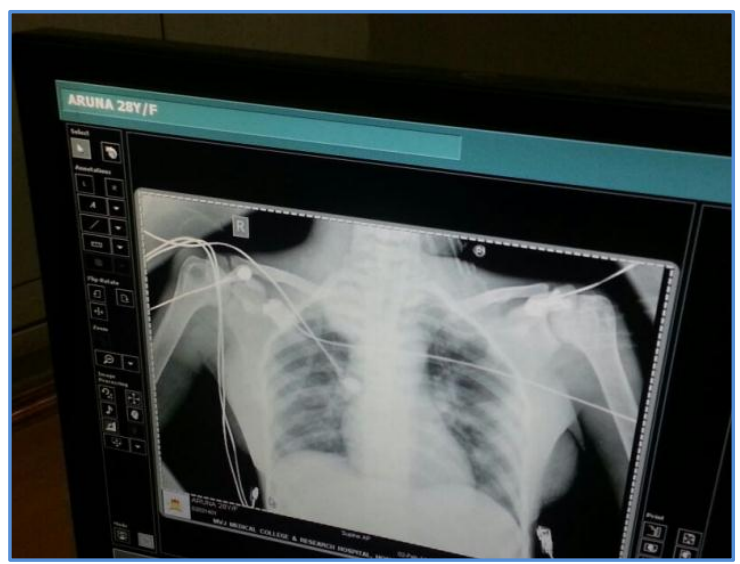

Fig. 1

\section{AUTHORS:}

1. Prasad Kulkarni

2. Naga Shiva Karthik Bhandaru

3. R. Shivaraman

\section{PARTICULARS OF CONTRIBUTORS:}

1. Professor, Department of Anaesthesiology, M.V.J. Medical College and Research Hospital, Hoskote, Bengaluru, Rajiv Gandhi University of Health Sciences, Karnataka, India.

2. First Year Post Graduate Student, Department of Anaesthesiology, M.V.J. Medical College and Research Hospital, Hoskote, Bengaluru, Rajiv Gandhi University of Health Sciences, Karnataka, India.

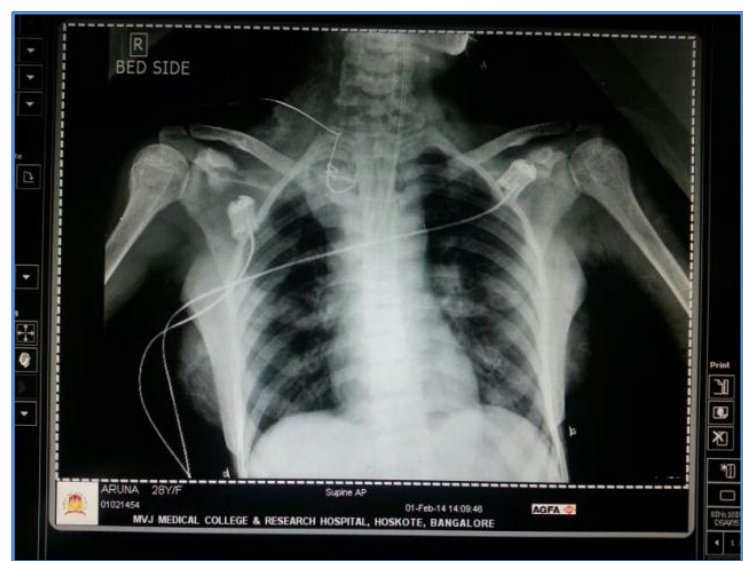

Fig. 2

3. Final Year Post Graduate Student, Department of Anaesthesiology, M.V.J. Medical College and Research Hospital, Hoskote, Bengaluru, Rajiv Gandhi University of Health Sciences, Karnataka, India.

\section{NAME ADDRESS EMAIL ID OF THE CORRESPONDING AUTHOR:}

Dr. Prasad Kulkarni,

G-5, Geethanjali Icon Apartment,

Nagavrpalya Main Road,

CV Raman Nagar,

Bangalore 560093.

Email:prasadkulk@yahoo.com

Date of Submission: 11/09/2014.

Date of Peer Review: 12/09/2014.

Date of Acceptance: 22/09/2014.

Date of Publishing: 29/09/2014. 